EPJ Web of Conferences 110,01030 (2016)

DOI: $10.1051 /$ epjconf/201611001030

(C) Owned by the authors, published by EDP Sciences, 2016

\title{
CONJUGATE HEAT TRANSFER DURING VISCOUS INCOMPRESSIBLE LIQUID MOVEMENT IN THE CAVITY CONSIDERING ITS COOLING THROUGH OUTER BOUNDARY
}

\author{
A.V. Krainov ${ }^{1, a}$, V.A. Arhipov ${ }^{2}$ \\ ${ }^{1}$ National Research Tomsk Polytechnic University, 634050 Tomsk, Russia \\ ${ }^{2}$ National Research Tomsk State University, 634050 Tomsk, Russian
}

\begin{abstract}
The paper presents the numerical simulations of the motion of a viscous incompressible non-isothermal fluid in an open rectangular cavity under conditions of conjugate heat transfer. The hydrodynamic flow pattern of a viscous fluid in the cavity obtained. The temperature profiles for the two phases - solid and liquid obtained. The influence of model parameters on the nature of motion and heat transfer conditions studied. Shows the effect of external cooling from the lateral surface on hydrodynamics and heat transfer of liquid in the cavity.
\end{abstract}

Important applications are the processes of heat transfer with consideration of convective flows. Such processes are realized in the devices of thermal power plants and systems. The necessity of studying the modes of motion environments and complex conditions of heat exchange associated with the development of such energy-intensive industries as energy, metallurgy, chemical and many others [18].

We study the process of movement of the molten metal in the recess with regard to heat transfer from its walls. The thermophysical properties of the melt and the cavity walls are identical. The aim of this work is to investigate the fluid flow and conjugate heat transfer during the motion of a viscous fluid in a rectangular cavity with consideration of the cooling of its outer border (lateral wall). Previously [9-19] have been solved a similar problem with the conditions of thermal insulation at the external borders of the cavity and the conditions of heat exchange with separate portions of the outer boundary of a cavity.

Modeling of heat transfer for the motion of a viscous fluid in the cavity associated with the solution of fairly complex problems, convective flows of incompressible fluid. Since the establishment of reliable analytical methods for calculating the parameters of non-isothermal flow of a viscous incompressible fluid in limited amounts of various types are excluded due to the complexity of such flows, there is a need of numerical simulation [20,21].

When formulating the problem, the following assumptions were made: 1) material of liquid and solid phases is identical; 2) thermal and physical parameters of liquid and wall material are taken identical; 3) laminar flow regime is realized; 4) possible effects of gas release, caused by chemical and physical processes, were not considered.

${ }^{a}$ Corresponding author: lux_veritatis@mail.ru 
Study of the described process were carried out using the Navier-Stokes equations, energy equation, heat conduction equation with appropriate initial and boundary conditions

$$
\begin{gathered}
\frac{\partial \omega}{\partial \tau}+U \frac{\partial \omega}{\partial X}+V \frac{\partial \omega}{\partial Y}=\frac{1}{\operatorname{Re}}\left(\frac{\partial^{2} \omega}{\partial X^{2}}+\frac{\partial^{2} \omega}{\partial Y^{2}}\right) \\
\frac{\partial^{2} \psi}{\partial X^{2}}+\frac{\partial^{2} \psi}{\partial Y^{2}}=\omega \\
\frac{\partial \theta}{\partial \tau}+U \frac{\partial \theta}{\partial X}+V \frac{\partial \theta}{\partial Y}=\frac{1}{\operatorname{Re} \cdot \operatorname{Pr}}\left(\frac{\partial^{2} \theta}{\partial X^{2}}+\frac{\partial^{2} \theta}{\partial Y^{2}}\right) \\
\frac{\partial^{2} \theta}{\partial X^{2}}+\frac{\partial^{2} \theta}{\partial Y^{2}}=\frac{\partial \theta}{\partial \mathrm{Fo}}
\end{gathered}
$$

Numerical solution of hydrodynamic task was carried out in area 2, limited land flowing 1, line of symmetry 3, the side wall 6 and the bottom of the cavity 4 and the region of the exit from the cavity 7 (Fig.1).

At the lower boundary $(y=S, D<x<L)$ and lateral $(x=D, S<y<H)$ the impermeability and adhesion conditions are specified, as well as boundary condition of the fourth kind for the energy equation.

The conditions of continuity of heat flow and nonpenetration are specified on the line of symmetry ( $x=L, S<y<H$ ). In the area of exit there were specified conditions of "drift" and "soft" boundary condition for velocity and temperature respectively [22].

On the outer border of the lateral surface of the cavity is set to a boundary condition of the third kind:

$$
\frac{\alpha_{1} \delta_{x}}{\lambda} \Delta \theta=-\frac{\partial \theta_{1}}{\partial X} .
$$

Thermal insulation condition is specified for the outer border of the bottom surface.

Here Fo - Fourier number; Re - Reynolds number; Pr - Prandtl number; $\theta$ - dimensionless temperature of the fluid; $\theta_{1}$-dimensionless temperature of the material cavity; $X, Y$-dimensionless Cartesian coordinates; $U, V$ - dimensionless longitudinal and transverse velocity component of the fluid motion, respectively; $\omega, \psi$ - variables vortex - stream function; $x, y$-dimensional Cartesian coordinates; $L, H$ - linear dimension of cavity along the $x$-coordinate and $y$ respectively; $D, S$ - linear dimension of cavity wall along coordinate $x$ and $y$, respectively; $\Delta \theta$ - dimensionless difference of temperature between the outer surface and the environment; $\alpha_{1}$ - heat-transfer coefficient; $\lambda$ - thermal conductivity coefficient; $\delta_{x}-$ wall thickness along the $x$-axis.

Equations solved by finite difference method [23, 24]. Difference analogs of transfer and heat conduction equations are solved using the sweep method $[25,26]$. The Poisson's equation for every sacrificial layer was solved using the successive over-relaxation method. Difference scheme of second order accuracy was used. The computations were performed on uniform and non-uniform difference grids. Assessment of the reliability of numerical simulation results was carried out by checking the conservativeness of difference schemes used a similar method was used in $[13,15]$. 
A different type of liquid (molten steel, molten light metals, etc.) was studied. In Fig.2 - 4 shows typical results of numerical studies for molten steel.

The analysis of the hydrodynamics of a flow allows to distinguish characteristic features of the behavior of a moving medium in the field of direct and reverse flow (Fig.2).

With the increase in speed of the flowing liquid profile of the transverse component of the velocity in the initial cross-section of the cavity becomes more filled and close to a constant value (the rate of filling decreases by $23 \%$ in comparison with the conditions of heat removal through the outer surface of the cavity).

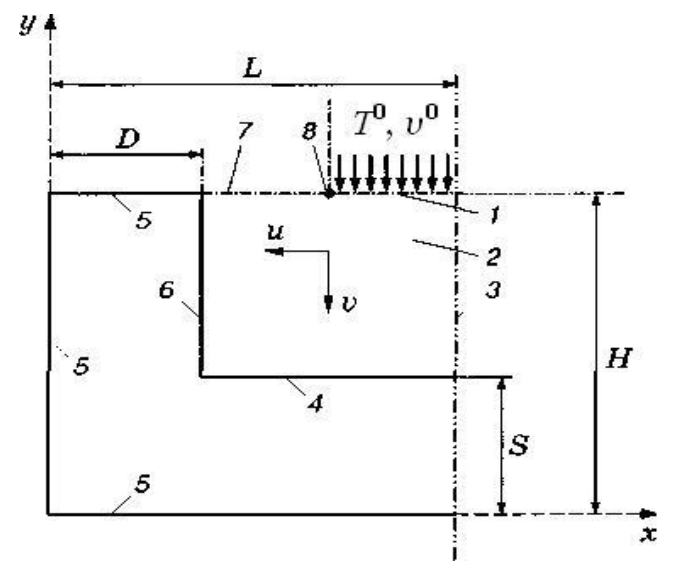

Figure 1. General diagram of flow in the cavity and geometry of computational domain (1-8): 1, 7 - permeable areas (areas of entrance and exit accordingly); 2 - hydrodynamic area; 3 - symmetry axis; 4,6 - inner surfaces; 5 - outer surfaces; 8 - boundary line between the permeable areas.

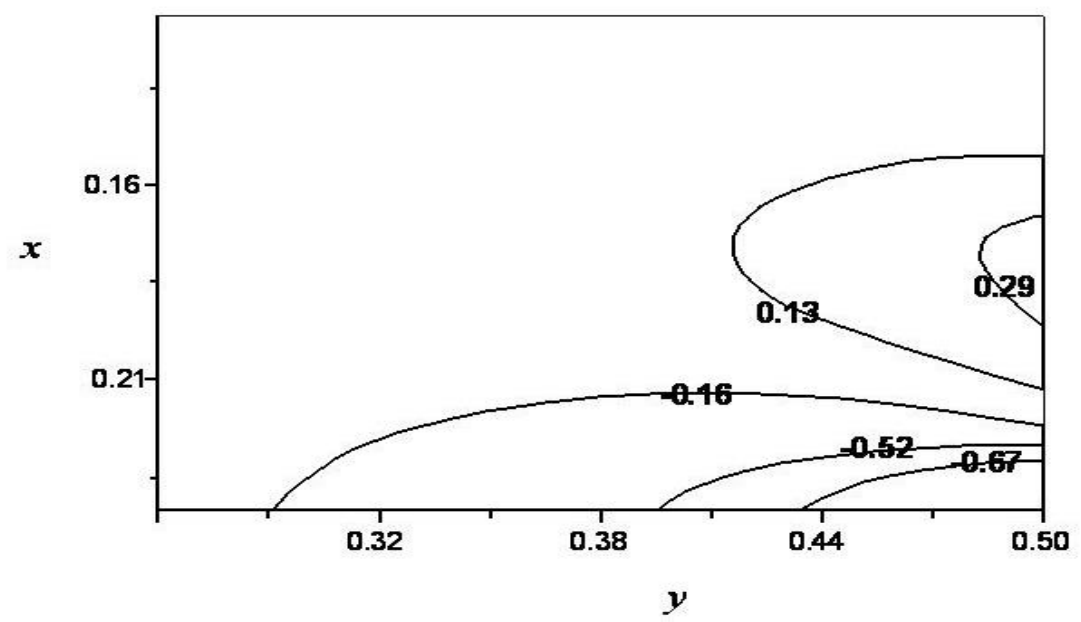

Figure 2. Isolines of velocity cross component at the time point $\mathrm{Fo}=4.5 \cdot 10^{-2}$ with the number $\mathrm{Re}=400$ and geometric ration of cavity sides $L / H=1 / 2$.

When liquid flows to the base of the cavity profile of the transverse component of the velocity starts to fall amid increasing longitudinal component (Fig.3). In the study of this process, it is necessary that the following integral relations that defines the fluid flow, which is the main integral characteristic of the flow: 


$$
\int_{x_{2}}^{x} v_{+}(x, y) d x=\int_{x}^{1} v_{0}(x, y) d x,
$$

where $x_{1}$-is the fixed point on the upstream section lying on axis of symmetry; $x_{0}$ - coordinate of the point of the division between plots with different direction of liquid flow in the cavity $\left(x_{2}<x_{0}<x_{1}\right)$; $x_{2}$ - coordinate extreme points of the fixed output section, lying on the side wall of the cavity; $v_{-}(x, y)-$ the transverse velocity component of fluid motion in the direction from the area of entrance to the bottom of the cavity; $v_{+}(x, y)$ - the transverse velocity component of fluid motion in the direction from the bottom of the cavity to the area of exit.

The distribution of longitudinal velocity component at the time point $\mathrm{Fo}=7,5 \cdot 10^{-2}$ with $\mathrm{Re}=400$ and geometric ratio of cavity sides $L / H=1 / 2$ are given in Fig.3. As follows from the hydrodynamics analysis in the motion of the fluid to the base of the excavation transverse speed decreases in conditions of growth of the longitudinal velocity component. On the return movement phase to the exit area the longitudinal velocity component decreases and the transverse velocity increases.

Distribution of temperatures in solid and liquid phases are given in Fig.4 in the plane $X, Y$ at the time point $4,7 \cdot 10^{-2}$ with $\operatorname{Re}=300, \operatorname{Pr}=0.979$. In the conditions of heat removal through the outer side surface of the cavity pattern of the temperature distribution in the liquid phase is significantly more pronounced than in the solid. This is because the transfer of heat in liquid phase is carried out not only by conduction but also by convection, and by heat removal through the external border of the lateral surface of the cavity. The temperature abruptly changes in the region $0.28<y<0.49,0.25<x<0.45$. In the hydrodynamic region the thermal profile becomes more filled with $0.22<y<0.4,0.21<x<0.45$.

With increasing entrance area temperature profile changes more gradually along the height of the cavity and the time to fill it near the inner bottom surface decreases and the inner lateral surface increases. This is due to the influence of the heat sink from the entire lateral surface of the outer contour of the cavity. In the case of reducing the value a of the temperature field is changing more dramatically at the height of the cavity and the filling profiles near the inner walls will increase significantly from the outer surface of the side wall of the cavity.

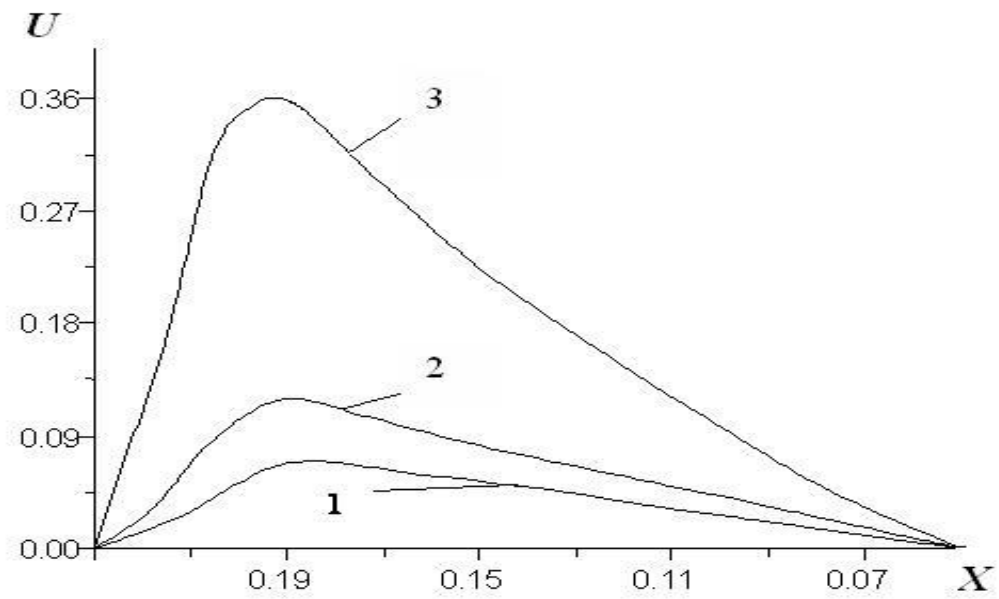

Figure 3. The distribution of longitudinal velocity component at the time point $\mathrm{Fo}=7,5 \cdot 10^{-2}$ with $\mathrm{Re}=400$ and geometric ratio of cavity sides $L / H=1 / 2$. The curves correspond to the values $U(X)$ in sections: $1-y=0.83 ; 2$ $y=0.75 ; 3-y=0.67$. 


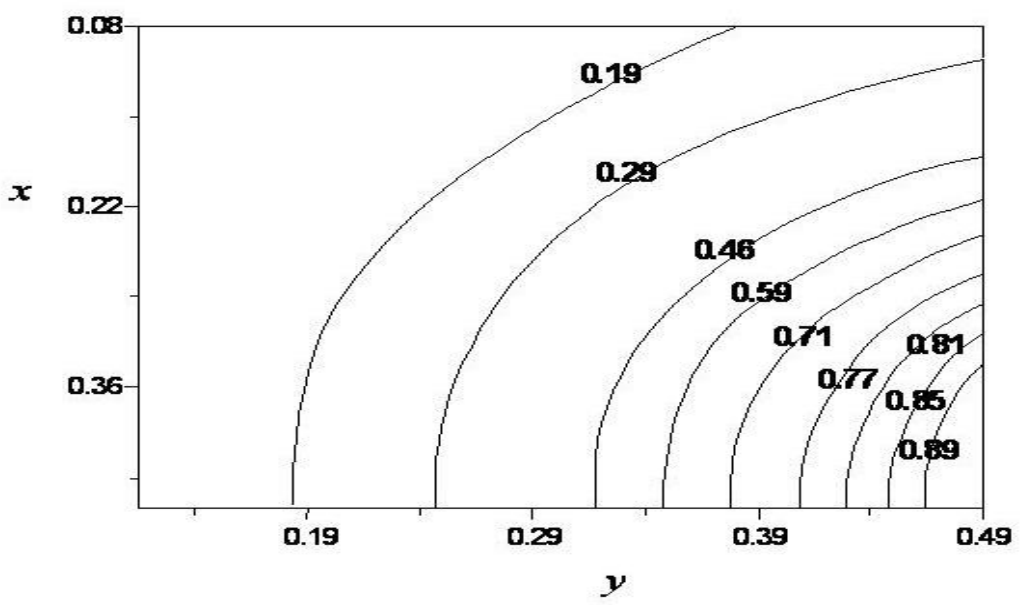

Figure 4. Distribution of temperatures in the plane $X, Y$ at the time point $4,7 \cdot 10^{-2}$ with $\operatorname{Re}=300, \operatorname{Pr}=0.979$.

The nature of the temperature distributions in the liquid and solid phases remains qualitatively with change of the dynamic parameters, the size of the input area and the geometrical relations. The range of temperature change in the conditions of external heat transfer for the considered processes is greatly increased, which has a considerable influence on the formation of patterns of fluid flow.

The results of numerical analysis allow to evaluate the effect of cooling the outer surface of the side wall of the cavity in terms of heat transfer and hydrodynamic pattern of the liquid flow.

The conducted researches allow to make a conclusion about the possibility of further expanding the area of application of mathematical apparatus [11, 12, 15-18] for solving problems of heat and mass transfer in the conjugate formulation for the motion of fluid in cavities of complex geometries.

The work was realized within the research state assignment "Science” №13.1339.2014/K (Code of Federal Target Scientific and Technical Program 2.1410.2014).

\section{References}

1. T.Cebeci, P.Bradshaw, Physical and Computational Aspects of Convective Heat Transfer (1987)

2. N.N. Rykalin, A.A. Uglov, L.M. Anischenko, High-Temperature production processes (Thermophysical basics, 1985)

3. G.V. Makhnova, V.V. Ris, E.M. Smirnov, Collection of the $2^{\text {nd }}$ National Heat and Mass Transfer Conference, 3, 100 (1998)

4. A.G. Fedorov, R. Viskanta, J. Heat Mass Transfer, 399 (2000)

5. V.Y. Polovnikov, A.V. Krainov, Heat Transfer (2012)

6. A.V. Krainov, Power engineering thermal techniques (2013)

7. G. Barakos, E. Mitsoulis, J. Numerical Methods in Fluids, 18, 695 (1994)

8. R. Janssen, R. Henkes, J. Fluid Mechanics, 209, 319 (1995)

9. O. Kymalainen, H. Tuomisto, O. Hongisto, Nuclear Engineering and Design, 149, 401 (1994)

10. A.V. Krainov, Proceedings of International Conference Conjugate problems of mechanics, computer science and ecology, 302 (2004)

11. A.V. Krainov, J. Izvestiya TPU, 306, 64 (2003)

12. A.V. Krainov, J. Izvestiya TPU, 306, 84 (2003)

13. G.V. Kuznetsov, M.A. Sheremet, J. Thermophysics and Aeromechanics, 12, 287 (2005) 
14. G.V. Kuznetsov, A.V. Krainov, G.V. Shvalova, J. In the world of scientific discoveries, 12, 119 (2010)

15. G.V. Kuznetsov, A.V. Krainov, J. Applied Mechanics and Technical Physics, 42, 851 (2001)

16. G.V. Kuznetsov, A.V. Krainov, J. High Temperature, 41, 252 (2003)

17. A.V. Kraynov, E. N. Pashkov, P.G. Yurovsky, J. Advanced Materials Research, 1040, 638 (2014)

18. A.V. Kraynov, G.V. Kuznetsov, EPJ Web of Conferences, 76, 1 (2014)

19. G.V. Kuznetsov, M.A. Sheremet, J. Thermophysics and Aeromechanics, 16, 119 (2009)

20. S. Armfield, Computers Fluids, 20, 1 (1991)

21. A. Lippke, H. Wagner, Computers Fluids, 20, 19 (1991)

22. P.J. Roache, Computational Fluid Dynamics (1980)

23. V.M. Paskonov, V.I. Polezhayev, L.A. Chudov, Numerical simulation of heat and mass transfer processes (1984)

24. E.L. Tarunin, Computational experiment in the problems on free convection (1990)

25. D. Shi, Numerical methods for solving heat transfer problems (1988)

26. A.A. Samarskiy, P.N. Vabischevitch, Computational heat transfer (2003) 